\title{
O USO DE TECNOLOGIA NO ENSINO E NA APRENDIZAGEM DE CÁLCULO DIFERENCIAL
}

Gisele Scremin ${ }^{1}$

Marli Teresinha Quartieri ${ }^{1}$

Eniz Conceição Oliveira ${ }^{1}$

Jorge Luis Palacios Felix ${ }^{2}$

\section{RESUMO}

Este trabalho apresenta o relato de experiência sobre a utilização de tecnologia no ensino e na aprendizagem dos conceitos de cálculo diferencial. Explorou-se a visualização gráfica do software Desmos em uma turma de 15 alunos, na disciplina de Cálculo I do curso de Agronomia de uma Instituição de Ensino Superior do Rio Grande do Sul. O objetivo foi verificar as contribuições do uso do Desmos para o ensino de derivadas de primeira e segunda ordem, e o comportamento de funções por meio do uso da visualização proporcionada pelo programa. Foram apresentadas atividades desenvolvidas com auxílio do Desmos para a compreensão dos conceitos das derivadas de primeira e segunda funções de uma variável real e o seu comportamento. Os resultados demonstram que o uso da visualização gráfica possibilita um caminho facilitador no ensino e na aprendizagem de conceitos abstratos da matemática, presentes em definições e teoremas, em especial no caso das derivadas.

Palavras-chave: Tecnologia. Desmos. Derivadas. Gráfico de funções.

Recebido em: 17/01/2018

Aprovado em: 24/04/2018

\footnotetext{
${ }^{1}$ Universidade do Vale do Taquari, UNIVATES, Lajeado, Rio Grande do Sul, Brasil.

2 Universidade Federal da Fronteira Sul, UFFS, Cerro Largo, Rio Grande do Sul, Brasil.
} 


\title{
THE TECHNOLOGY APPLICATION IN THE TEACHING LEARNING OF DIFFERENTIAL CALCULUS
}

\author{
Gisele Scremin \\ Marli Teresinha Quartieri \\ Eniz Conceição Oliveira \\ Jorge Luis Palacios Felix
}

\section{ABSTRACT}

This paper presents the experience report of the technology used in the teaching-learning of the concepts of differential calculus. The graphic display of the software Desmos was explored in a Calculus I class of fifteen students of the Agronomy Degree in a Institution of Higher Education of Rio Grande do Sul, with the goal to verify the contributions of Desmos to the teaching of derivatives of the first and second order and the function performance through the application of the view proportioned by the software. Activities developed with the assistance of Desmos are presented to achieve the comprehension of the concepts of the derivatives of first and second function of a real variable and its performance. The results demonstrate that the application of the graphic view opens a enabler way in teaching and learning of abstract concepts of mathematics, which are related to the definitions and theorems specially in the case of derivatives.

Keywords: Technology. Desmos. Derivatives. Functions graphing. 


\section{INTRODUÇÃO}

O Cálculo Diferencial e Integral é uma disciplina presente no currículo de muitos cursos de Ensino Superior, tanto por ser um ramo importante da matemática quanto por seu campo de aplicações estender-se a outras áreas de conhecimento. Apesar das aplicabilidades da disciplina, as dificuldades nos processos de ensino e aprendizagem vêm sendo foco de muitas pesquisas, como as de Junior, Bessa e Cezana (2015), Rafael e Escher (2015), Alvarenga, Dorr e Vieira (2016), uma vez que ela é alvo de um elevado número de reprovações, evasão e repetência. Algumas tendências na Educação Matemática têm sido propostas na tentativa de minimizar os efeitos dessa problemática, tais como a utilização de Recursos Tecnológicos, a Modelagem Matemática e a Resolução de Problemas. De acordo com Silva (1994, p. 6), esses são alguns dos "caminhos que se pode ensejar melhor produtividade no processo de ensino e aprendizagem de Cálculo Diferencial e Integral" e que vêm demonstrando alguns resultados positivos, como alunos mais ativos e participativos (PONTE; BROCARDO; OLIVEIRA, 2006), e melhor desempenho na resolução de problemas (NASCIMENTO; LOPES; TEIXEIRA, 2013). Entre as tendências apontadas, optou-se por analisar as contribuições do uso dos recursos tecnológicos como estratégia para o ensino e a aprendizagem do Cálculo Diferencial, especificamente o uso do software Desmos para o ensino de derivadas de primeira e segunda ordem e o comportamento de funções. Assim, estando em meio a essa revolução tecnológica, em que os alunos são considerados nativos digitais, faz-se necessário conhecer e aproveitar a tecnologia para contribuir na construção do conhecimento.

O uso de tecnologias, segundo Junior (2015), tem proporcionado oportunidades de observar e experimentar o que está acontecendo com certos fenômenos, possibilitando a visualização e a múltipla representação das informações. Neste trabalho, aborda-se a visualização que é proporcionada pelo uso do computador, mais especificamente pelo uso do software Desmos, que é um programa gráfico que possibilita a construção de curvas, tabelas, marcação de pontos, adição de controle deslizante, entre outras funções. Trata-se de um programa multi-idioma, que pode ser acessado de qualquer aparelho, e possui uma interface amigável e de fácil acesso, podendo ser utilizado para trabalhos colaborativos, desde que seja realizado um cadastro de usuário.

A visualização gráfica proporcionada pelos softwares é parte importante no processo de ensino e aprendizagem, principalmente na área da Matemática, pois permite imbricar conceitos e significados que podem ser facilmente incorporados à solução simbólica de problemas ou ao conteúdo. A visualização tem um poderoso papel complementar, em que se pode destacar três aspectos: como apoio a resultados essencialmente simbólicos; como possibilidade para resolver conflitos entre soluções simbólicas e intuições; como ajuda a 
reengajar e a recuperar os fundamentos conceituais que podem ser facilmente contornados por soluções formais (ARCAVI, 2003). O ensino e a aprendizagem, segundo o referido autor, necessitam propiciar formas para que se possa melhor ver os conceitos matemáticos, por meio da exploração da visualização em sua totalidade, que, além de contribuir na organização dos dados, é um importante fator na condução do desenvolvimento analítico da solução. A visualização, portanto, pode ser considerada como parte integrante do próprio processo analítico da solução.

Quanto ao professor em frente do uso de tecnologias, Costa e Salvador (2004) salientam que o docente tem o papel de mediador e orientador, contribuindo para que o aluno seja capaz de realizar uma análise crítica e construir o conhecimento, e não somente contemplar a beleza gráfica e o mundo colorido que o computador oferece. O professor, então, é mais importante que nunca, pois ele precisa se apropriar dessa tecnologia e introduzi-la na sala de aula, além de começar a lidar com o conhecimento de modo diferente. Isso, segundo Andrade (2003, p. 82), porque "as tecnologias não são nada sem as pessoas com capacidade de poder retirar delas os refinamentos da qualidade, e assim realizar uma orientação nova e uma visão criativa", ressaltando-se, dessa forma, a importância do preparo e da adequação do professor para tal tarefa tão importante.

Com a intenção de abranger essa perspectiva, o presente trabalho se configura em um relato de experiência referente à intervenção pedagógica realizada como demanda da disciplina de Estágio e Pesquisa Supervisionada, do Curso de Pós-graduação em Ensino de Ciências Exatas da Universidade do Vale do Taquari (Univates). Essa interferência pedagógica no processo de ensino e aprendizagem da Matemática teve por objetivo a utilização de atividades exploratórias sobre comportamento gráfico das funções polinomiais e suas derivadas, com utilização do software Desmos, para o estudo desse comportamento e suas variações.

\section{A VISUALIZAÇÃO COMO PARTE DO PROCESSO DE ENSINO E APRENDIZAGEM}

O progresso tecnológico impulsionou e modificou as formas de ensinar e aprender. Os alunos da atualidade representam a nova geração que cresceu com as novas tecnologias, que desde muito cedo utiliza telefones, tablets, computadores, tem acesso ao mundo de informações, jogos, sites, entretenimento, entre outros. Essa realidade de interação tecnológica antecipada pode ser utilizada em benefício da educação, seja ela na Educação Básica ou no Ensino Superior. Metodologias de ensino que integrem o uso de tecnologias podem favorecer a aprendizagem e ainda possibilitar um ambiente mais dinâmico e motivador. 
Pesquisas em Educação Matemática relacionadas ao uso de tecnologias vêm surgindo de forma gradativa, entre elas podemos destacar Allevato (2010), Borba e Villarreal (2005), entre outros. Esses autores concordam que o uso de softwares computacionais possibilita uma inovação no ensino, pois são considerados ferramentas auxiliares na construção de conceitos e aplicações relacionados ao ensino de Matemática. Segundo Borba e Penteado (2001), a utilização de software possibilita a experimentação em conteúdos matemáticos, além de estimular a percepção visual dos alunos. Os autores salientam que as possibilidades experimentais das tecnologias podem ser exploradas, podendo-se chegar à elaboração e à validação de conjecturas, além de conduzir os alunos a desenvolverem suas ideias e a elaborarem novas conjecturas. Porquanto, alunos, quando em contato com tecnologias, tornam-se investigativos e participativos, e não apenas receptivos.

Dessa forma, vemos que o contexto educacional vem se transformando e precisa atender a novas expectativas do ensino e da aprendizagem. Nos últimos anos, os recursos utilizados tendem a ir além da sala de aula e do uso do livro didático. Nesse contexto, o emprego da tecnologia surge, entre outras, como uma das tendências atuais para superar o quadro de reprovação, abandono e repetição dos discentes de alguns cursos de Cálculo, pois, para muitos, aprender Matemática parece não ser uma tarefa tão fácil. Uma das razões pelas quais a Matemática pode ser difícil de aprender está no fato de que os conceitos dessa disciplina (por exemplo, números e funções) não são intuitivos ou acessados por meio da experiência diária (CHIAPPINI; BOTTINO, 1999). Assim, o uso de visualizações consiste em uma maneira de facilitar a aprendizagem de Matemática.

Pesquisas sobre visualização na aprendizagem de Matemática, segundo Presmeg (2006), iniciaram-se lentamente, crescendo de uma base psicológica no final da década de 1970 e início dos anos de 1980. Tais estudos estão relacionados a diversos ramos dessa área e são multifacetados, envolvendo aspectos históricos, filosóficos, psicológicos, pedagógicos e tecnológicos importantes (ZIMMERMANN; CUNNINGHAM, 1991). Esses estudos ainda vêm sendo desenvolvidos, tanto no âmbito da Educação Básica quanto do Ensino Superior, devido a sua importância nesse processo. Algumas dessas investigações apontam a visualização nessa área como um componente-chave do raciocínio na resolução de problemas e atividades, bem como para fins ilustrativos (ARCAVI, 2003). Além disso, de acordo com Rösken e Rolka (2006), essa é considerada uma ferramenta poderosa para explorar e dar significado aos conceitos matemáticos e à relação entre eles.

O termo visualização, para Zimmermann e Cunningham (1991), é empregado para descrever os processos de produção ou o uso de representações geométricas ou gráficas de conceitos, princípios ou problemas matemáticos, sejam desenhadas à mão ou geradas por computador. Ainda, Fainguelernt (1999) considerava a visualização como uma habilidade de 
perceber, transformar, descobrir, gerar, comunicar, documentar e refletir sobre as informações visuais. Partindo dessa definição, Santos (2009) destaca que o contato visual físico é necessário para que ocorra ou se promova o contato mental de um indivíduo. No contexto da Educação Matemática, fazer uso da visualização, além de promover a intuição e o entendimento, possibilita uma abrangência maior da cobertura em assuntos matemáticos, permitindo aos alunos não somente aprenderem matemática, mas se tornarem capazes de fazer sua própria matemática (FLORES; WAGNER; BURATTO, 2012). A visualização e a leitura de informações gráficas em Matemática são aspectos importantes, pois auxiliam a compreensão de conceitos e o desenvolvimento de capacidades de expressão gráfica (BRASIL, 1998).

Diante de diversas definições do termo, tanto no âmbito da Matemática quanto da Educação Matemática, pode-se perceber que a visualização está focada na percepção e na manipulação de imagens visuais, e que a aprendizagem é resultado dessa interpretação dada a sensações e estímulos do meio ambiente, a ideias, imagens, expectativas e atitudes (FAINGUELERNT, 1999). Entre os trabalhos que utilizaram o processo de visualização para a aprendizagem de conceitos matemáticos, pode-se mencionar Junior (2015), que relata a utilização do software Geogebra para o ensino de conceitos relacionados à derivada com um grupo de professores do Ensino Superior, desenvolvendo atividades exploratórias de construção e interpretação de gráficos. Os resultados obtidos apontam que a visualização proporcionada pelo software contribuiu para uma ressignificação de diversos conceitos e propriedades de derivadas que são requisitados na construção de gráficos de funções reais. Além disso, destacam a visualização como fundamental no ensino e na aprendizagem de Cálculo I, um equilíbrio entre os processos visuais e os processos algébricos.

\section{METODOLOGIA}

As atividades exploratórias foram desenvolvidas no período de oito horas/aula, correspondente a dois dias de trabalho (5 e 12 de maio de 2017) no período da noite, com alunos do Curso de Agronomia de uma Universidade Federal do Rio Grande do Sul.

O software Desmos ${ }^{3}$ abre-se em uma janela de trabalho, como mostra a Figura 1. Essa janela apresenta duas colunas: a coluna da esquerda é utilizada para inserir as fórmulas ou equações matemáticas (pode-se inserir expressão, nota, tabela, pasta e imagem), e a da direita mostra a saída gráfica sobre uma malha quadriculada ou plano cartesiano. Além disso, pode-se dispor de uma calculadora na própria tela. A seleção desse recurso ocorreu pela facilidade de compreensão dos comandos a serem utilizados, pela ótima saída de dados (gráfico) e pela possibilidade de exploração de aspectos considerados relevantes para

\footnotetext{
${ }^{3}$ O programa está disponível em: <https://www.desmos.com/>. Acesso em: 27 ago. 2018.
} 
este trabalho. Como principais características desse recurso, pode-se apontar: ser uma ferramenta on-line e de acesso gratuito; ser acessado por qualquer navegador de internet e por qualquer computador, tablet ou smartphone, desde que esteja conectado, sem a necessidade de sua instalação no dispositivo; não requer cadastro de usuário para ser utilizado, no entanto possui a opção de cadastro de usuário do Google, o que possibilita salvar e compartilhar as atividades realizadas; ser multi-idioma; funcionar como uma plataforma de trabalho colaborativo, ou seja, permitir o compartilhamento das atividades, as quais podem ser utilizadas por outros usuários.

Figura 1 - Janela de trabalho do Desmos

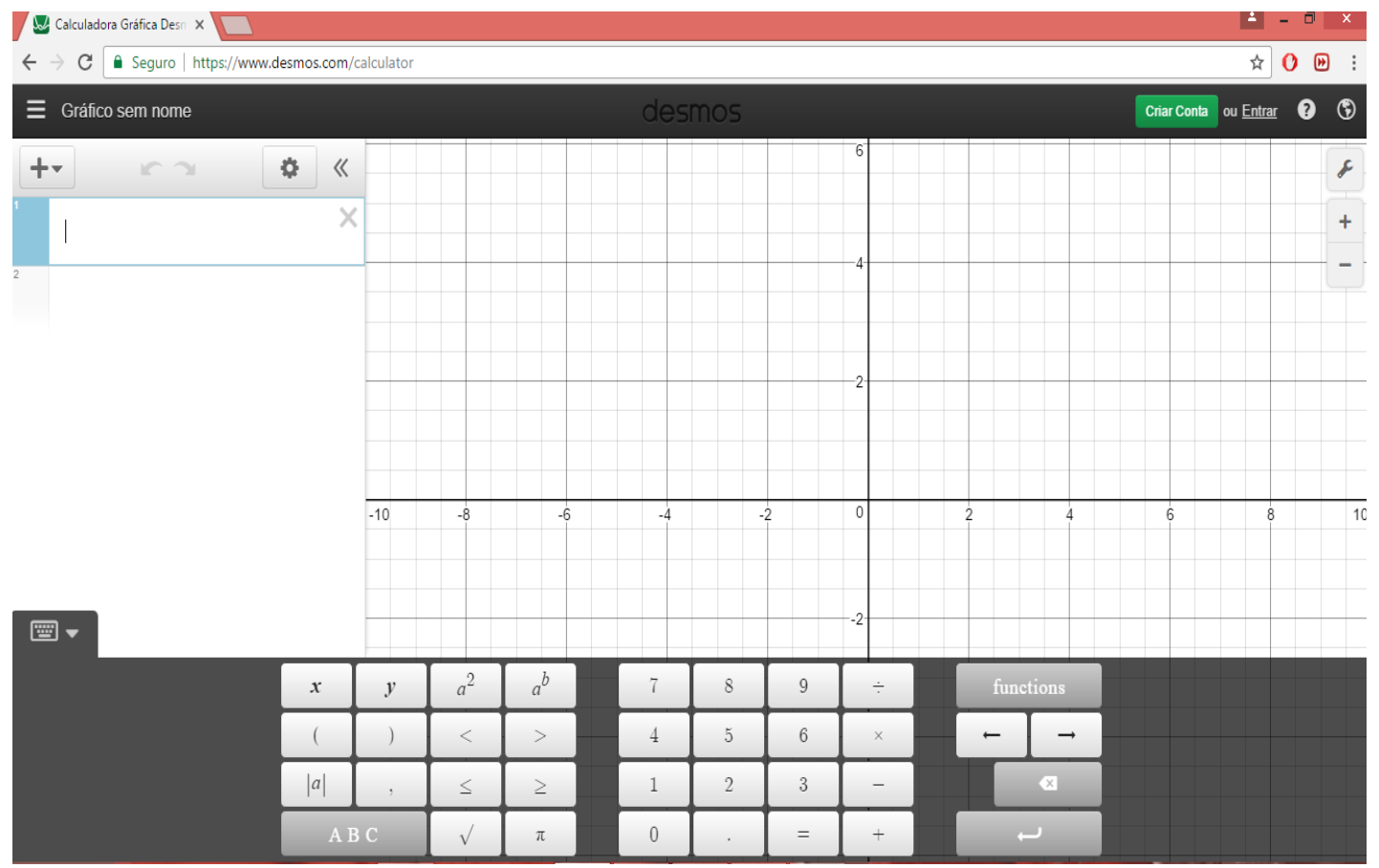

Fonte: elaborada pelos autores, 2017.

Para o desenvolvimento das atividades exploratórias, com intuito de facilitar a visualização dos gráficos construídos e analisados, foi solicitado aos alunos que trouxessem seus notebooks. Assim, com o software à disposição, as atividades foram desenvolvidas em duplas, o que proporcionou maior interação entre os alunos e entre os alunos e a professora, possibilitando um diálogo aberto e uma discussão sobre os conceitos envolvidos. As atividades do primeiro dia de aula elencaram como conteúdo a análise de uma função polinomial, por meio do gráfico de sua derivada primeira, destacando seus intervalos de crescimento e decrescimento e seus pontos críticos (Máximos e Mínimos). Ressalta-se que, em todo esse processo de análise dos gráficos, foi utilizado o aspecto algébrico e numérico para desenvolver os devidos cálculos, a fim de proporcionar um 
envolvimento das diversas abordagens. No Quadro 1 são apresentadas as atividades exploratórias do primeiro dia de aula:

Quadro 1 - Atividades exploratórias para análise da função e sua derivada primeira

\section{Crescimento e Decrescimento de uma Função e Pontos Críticos}

(Análise da Derivada Primeira)

1) Construa o gráfico da seguinte função: $f(x)=x^{3}+3 x^{2}+2$ usando o Desmos.

2) Calcule os valores de y para a função $f(x)$. Construa uma tabela com o Desmos com os seguintes valores para $x:-3,-2.75,-2.5,-2.25,-2$. Compare os resultados, o que acontece com os valores de $f(x)$ ?

3) Construa outra tabela com o Desmos, agora com os seguintes valores para $x:-2,-1.5,-1,-0.75$, 0.50 . Compare os resultados: o que acontece com os valores de $f(x)$ ?

4) Construa mais uma tabela com o Desmos, com os seguintes valores para $x: 0.5,0.75,1,1.25$. Compare os resultados: o que acontece com os valores de $f(x)$ ?

5) Por meio dos resultados obtidos anteriormente, e observando o gráfico da função, quando podemos dizer que uma função é crescente? E decrescente?

6) Agora vamos traçar o gráfico da derivada de $f(x)$, denotado por $g(x)$. Utilize o seguinte comando: $g(x)=\frac{d}{d x} f(x)$

7) Calcule os valores de y para a função $g(x)$. Construa uma tabela, utilizando o Desmos, com os seguintes valores para $x$ em $g(x):-3,-2.75,-2.5,-2.25$. Os valores encontrados são positivos ou negativos?

8) Construa outra tabela com o Desmos, agora com os seguintes valores para $x$ em $g(x)$ : -2, -1.5, -

$1,-0.75,-0.50$. Os valores encontrados são positivos ou negativos?

9) Construa mais uma tabela com o Desmos agora com os seguintes valores para $x \mathrm{em}$ $g(x): 0,0.5,0.75,1$. Os valores encontrados são positivos ou negativos?

10) Identifique os intervalos onde o gráfico de $g(x)$ é positivo. Faça o mesmo onde o intervalo é negativo. Compare o resultado com o crescimento e decrescimento de $f(x)$.

11) Como podemos denotar o crescimento e decrescimento da função por meio da sua derivada primeira?

12) Se fizermos $\frac{d}{d x} f(x)=0$ que valores encontramos? Observe o gráfico de $f(x)$ e marque os pontos encontrados. O que acontece com a $f(x)$ nesses referidos pontos?

13) Calcule o coeficiente angular da reta que passa nesses pontos. Qual o seu valor?

14) Trace as retas encontradas. Qual a posição dessas retas em relação ao eixo $x$ ?

15) Selecione valores para $x$ antes e depois dos pontos -2 e 0 . 0 que acontece com a imagem antes e depois desses pontos?

Fonte: elaborado pelos autores, 2017. 
No segundo dia de aula, foram exploradas algumas atividades com a finalidade de analisar o gráfico da segunda derivada e da função original e, também, relacioná-lo com a concavidade da função. Os alunos novamente realizaram as atividades com auxílio do software Desmos, em dupla. No Quadro 2 estão as atividades desenvolvidas.

Quadro 2 - Atividades sobre a concavidade da função polinomial através da derivada segunda

\section{Atividades de análise da concavidade da função por meio da derivada segunda}

1) Construa o gráfico da função $f(x)=x^{3}-3 x^{2}+1$ usando o software Desmos.

2) Construa o gráfico da derivada segunda, denotada por $h(x)$.

3) Em que intervalo o gráfico da $h(x)$ é positivo? E negativo?

4) Identifique o valor de $x$ quando $f^{\prime \prime}(x)=0$. Calcule a imagem de $x$ usando $f(x)$. Marque esse ponto no gráfico $(x, f(x))$.

5) O que acontece com a concavidade da $f(x)$ ao redor desse ponto (calculada no item 4)?

6) Qual a relação entre a concavidade da função e sua derivada segunda?

Fonte: elaborado pelos autores, 2017.

Na conclusão das atividades, aplicou-se um questionário aberto para elencar a opinião dos alunos ante a utilização do recurso tecnológico nas aulas de Cálculo, bem como saber sobre as contribuições do uso do software Desmos na compreensão do conteúdo de comportamento de uma função pela análise de sua derivada. As questões elencadas no questionário estão relacionadas no Quadro 3.

Quadro 3-Questionário sobre a utilização do Desmos

\section{Questionário sobre a utilização do software Desmos}

1) A utilização do software Desmos contribuiu para a compreensão dos conteúdos abordados? Por quê?

( ) Sim ( ) Não ( ) Um pouco

2) Teve alguma dificuldade ao utilizar o software? Qual?

3) Já utilizou algum software antes? Qual?

4) Considera importante o uso de tecnologias no ensino de Cálculo? Por quê?

Fonte: elaborado pelos autores, 2017.

\section{RESULTADOS E DISCUSSÕES}

O desenvolvimento das atividades exploratórias do Quadro 1 (dos itens 1 até 5) objetivaram compreender o comportamento de uma função polinomial em sua forma crescente e 
decrescente, sem usar derivadas a priori, por meio da análise dos pontos em uma tabela de valores e marcação dos pontos obtidos na curva da função (FIGURA 2), de modo que os alunos comprovassem a relação matemática expressa pela seguinte definição (STEWART, 2017): (a) Uma função $f$ é crescente em um intervalo $I$ se $f\left(x_{1}\right)<f\left(x_{2}\right)$ quando $x_{1}<x_{2}$ em $I$; (b) Uma função $f$ é decrescente em um intervalo $I$ se $f\left(x_{1}\right)>f\left(x_{2}\right)$ quando $x_{1}<x_{2}$ em $I$. A visualização gráfica permite verificar simultaneamente que, para um certo intervalo de valores de $x$, os valores de $f(x)$, na tabela mostrada na parte superior da Figura 2, crescem enquanto os pontos posicionam-se no gráfico da função, determinando um intervalo no qual a função é crescente, assim como os demais valores de $x$, apresentados na tabela inferior da Figura 2, originaram imagens que demonstram 0 decrescimento da função.

Figura 2 - Estudo de crescimento e decrescimento da função original $f(x)$
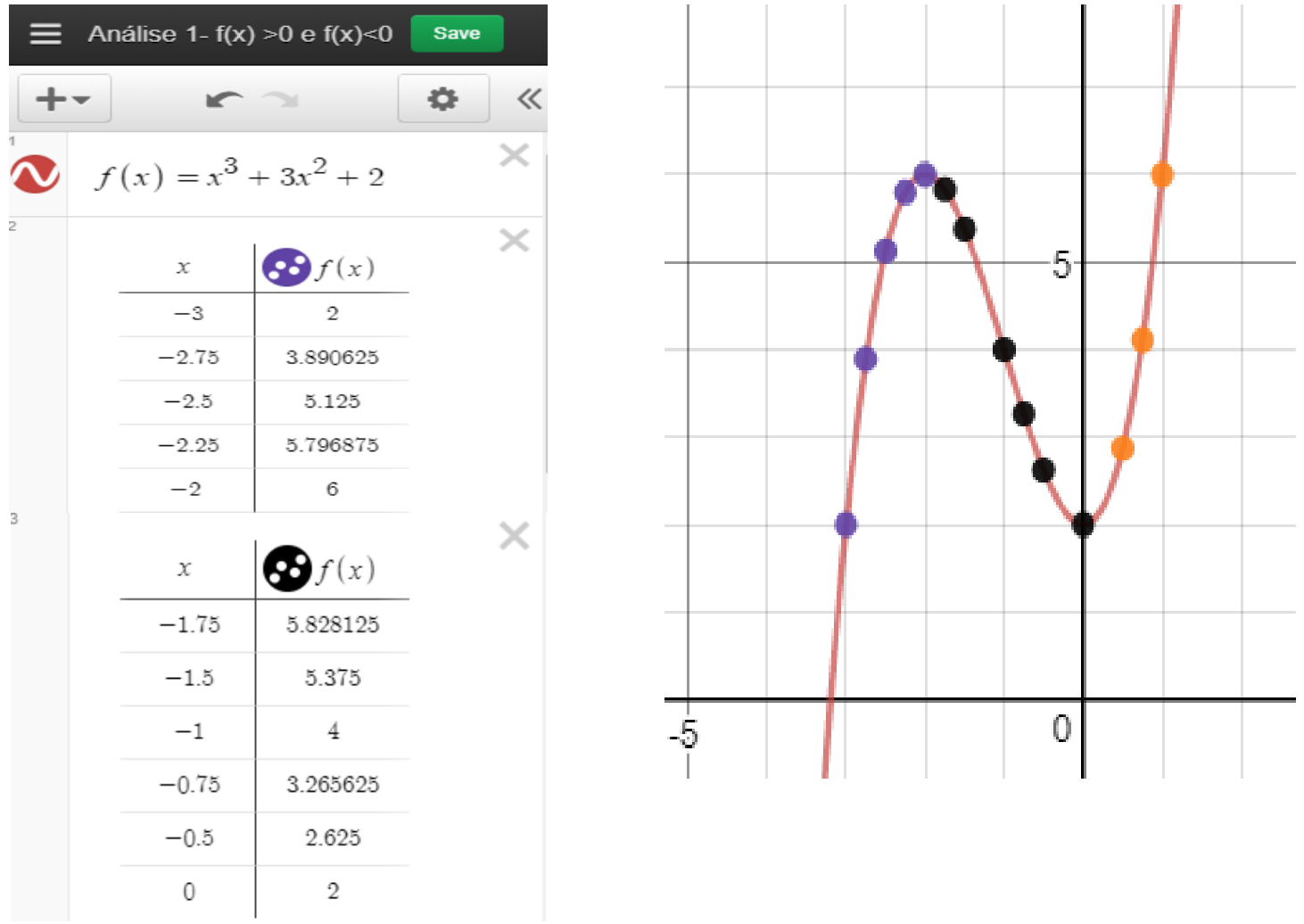

Fonte: elaborada pelos autores, 2017.

Na sequência, solicitou-se aos alunos o traçado do gráfico da derivada da função polinomial e a construção da tabela de valores para determinados intervalos, sugeridos pelas atividades dos itens de 6 a 11 do Quadro 1, como mostra a Figura 3. O intuito foi realizar a análise e a comparação do gráfico da função original com o gráfico da derivada primeira, de modo que pudessem verificar as seguintes relações matemáticas que definem 0 
comportamento da função em relação à derivada primeira (STEWART, 2017): Seja $f(x)$ uma função contínua em um intervalo fechado $[a, b]$ e diferenciável no intervalo aberto (a, b). Se $f^{\prime}(x)>0$ para todo valor de $x$ em $(\mathrm{a}, \mathrm{b})$, então $f$ é crescente em $[a, b]$. Se $f^{\prime}(x)<0$ para todo valor de $x$ em $(\mathrm{a}, \mathrm{b})$, então $f$ é decrescente em $[a, b]$. Se $f(x)=0$ para valor de $x$ em $(\mathrm{a}, \mathrm{b})$, então $f$ é constante em $[a, b]$.

Figura 3 - Gráfico da Função e de sua derivada primeira
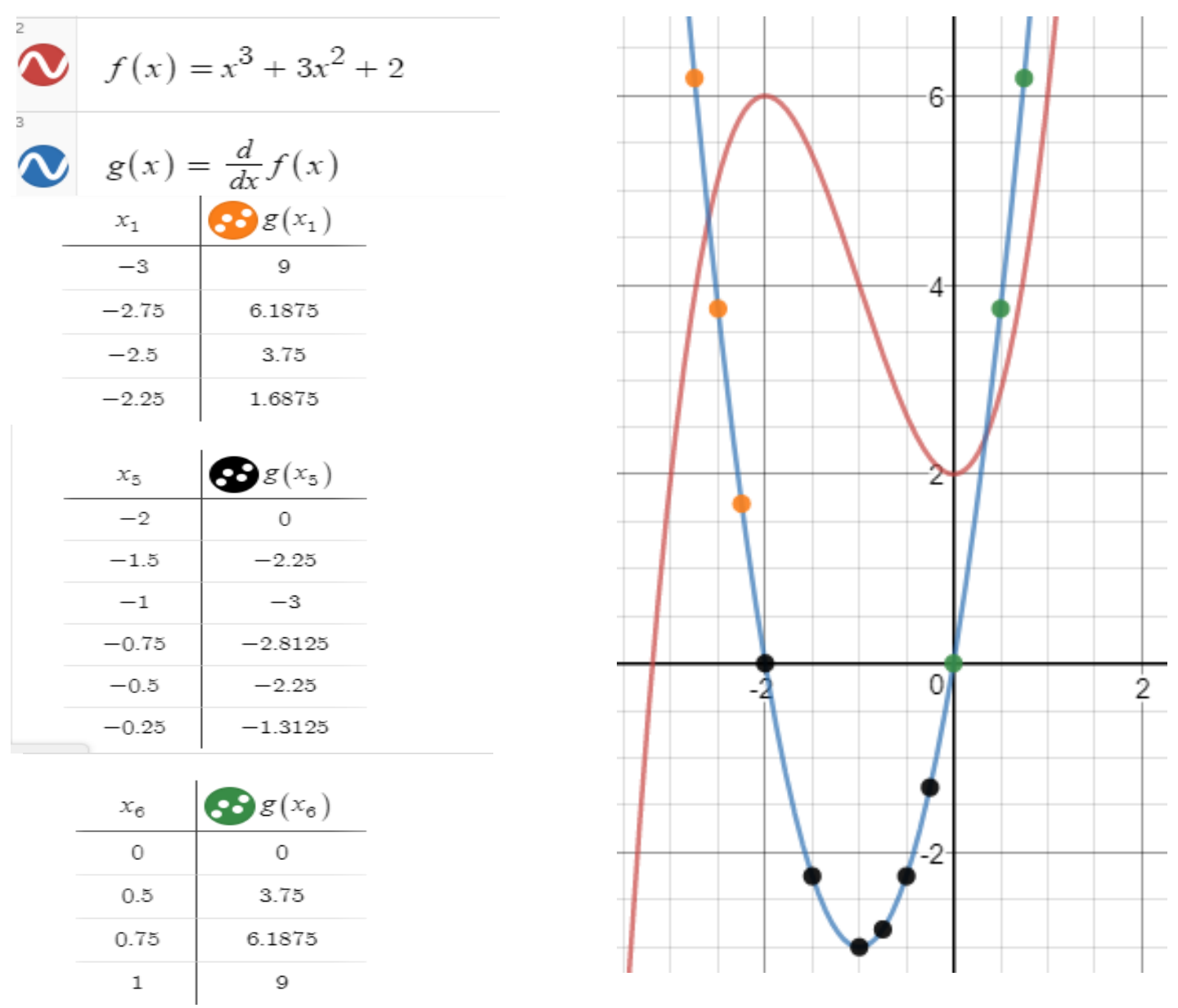

Fonte: elaborada pelos autores, 2017.

As atividades, dos itens de 12 a 15 do Quadro 1, foram propostas com o objetivo de reconhecer os pontos críticos de uma função e entender o motivo pelo qual é igualada a zero a função derivada para o cálculo desses pontos, além da necessidade de análise em torno deles para definir se são pontos de máximo ou de mínimo. Por meio dos gráficos (FIGURA 3) e dos cálculos realizados, as seguintes relações matemáticas puderam ser analisadas pelos alunos. Se uma função tiver um máximo ou um mínimo em $x=c$, então $c$ é um ponto crítico de $f$ que precisa ser analisado. Se o sinal da derivada for positivo à esquerda do ponto crítico e negativo à direita dele, o ponto é um máximo relativo; se o sinal 
da derivada for negativo à esquerda do ponto crítico e positivo à direita dele, o ponto é um mínimo relativo; se o sinal da derivada for o mesmo em ambos os lados do ponto crítico, o ponto não é máximo nem mínimo relativo.

Pode-se inferir que o aspecto visual proporcionado pelo software Desmos contribuiu com as atividades, pois oportunizou aos alunos a descoberta e a análise de alguns resultados obtidos numericamente, como pode ser observado na Figura 4. Nela, o aluno A respondeu às atividades propostas por meio da visualização e da interpretação dos gráficos da Figura 3.

Figura 4 - Respostas das atividades do aluno $\mathrm{A}$

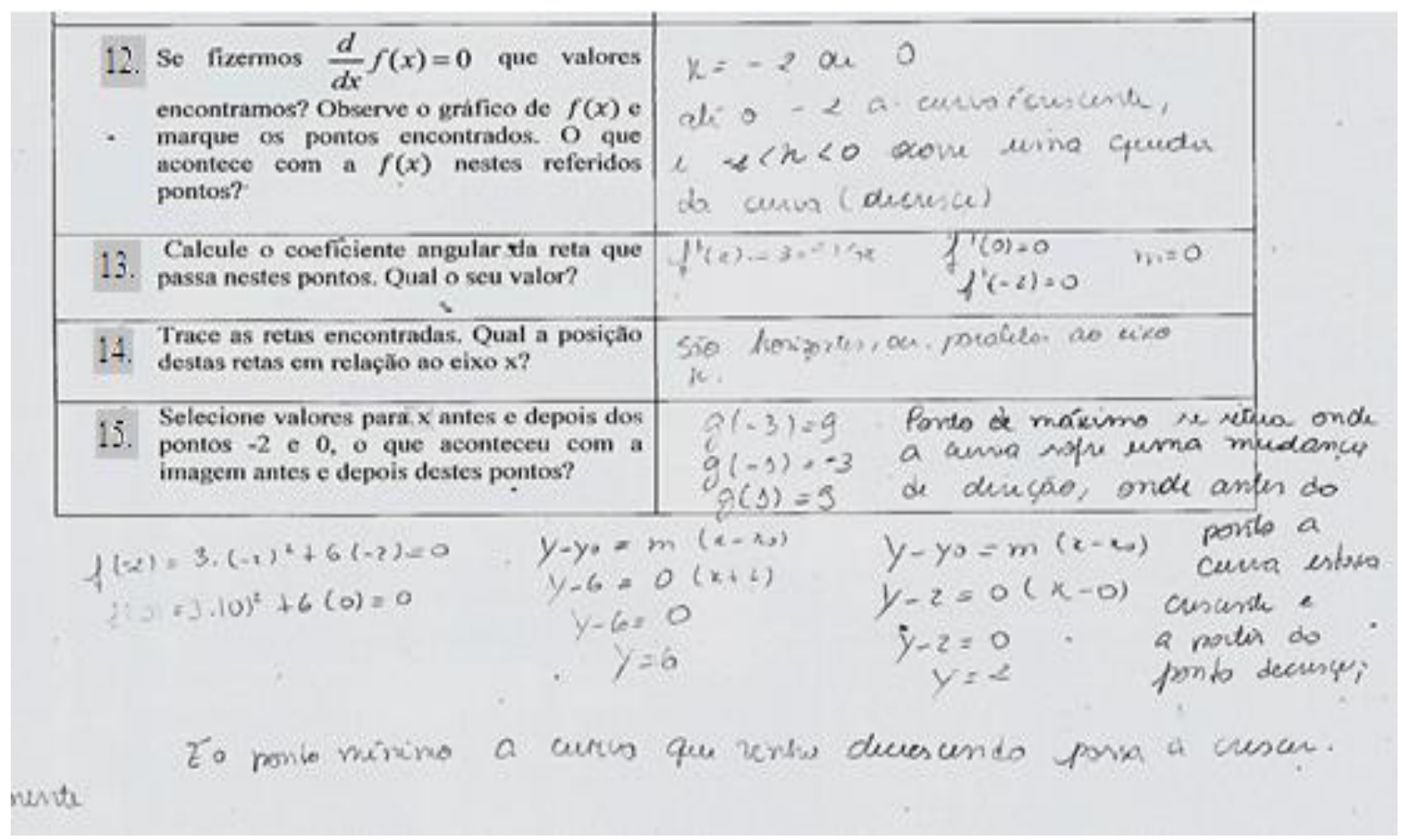

Fonte: atividades do aluno $\mathrm{A}$.

As atividades exploratórias referentes ao Quadro 2 foram realizadas para estabelecer a relação entre a derivada segunda da função e sua concavidade, bem como identificar o ponto de inflexão, ou seja, aquele ponto em que ocorre a mudança de concavidade da função. Para tal finalidade, foram construídos os gráficos da função original $f$ e da função derivada segunda $h=f^{\prime \prime}$ como mostra a Figura 5. Ademais, puderam ser analisados pelos alunos os seguintes princípios (STEWART, 2017): para uma função $f$ derivável até segunda ordem em um intervalo $I$, tal que $f^{\prime \prime}\left(x_{0}\right) \neq 0$, então (a) quando $f^{\prime \prime}\left(x_{0}\right)>0$, o gráfico de $f$ tem concavidade voltada para cima em $x_{0}$; (b) quando $f^{\prime \prime}\left(x_{0}\right)<0$, o gráfico de $f$ tem concavidade voltada para baixo em $x_{0}$. 
Figura 5 - Gráfico da Função e de sua derivada segunda
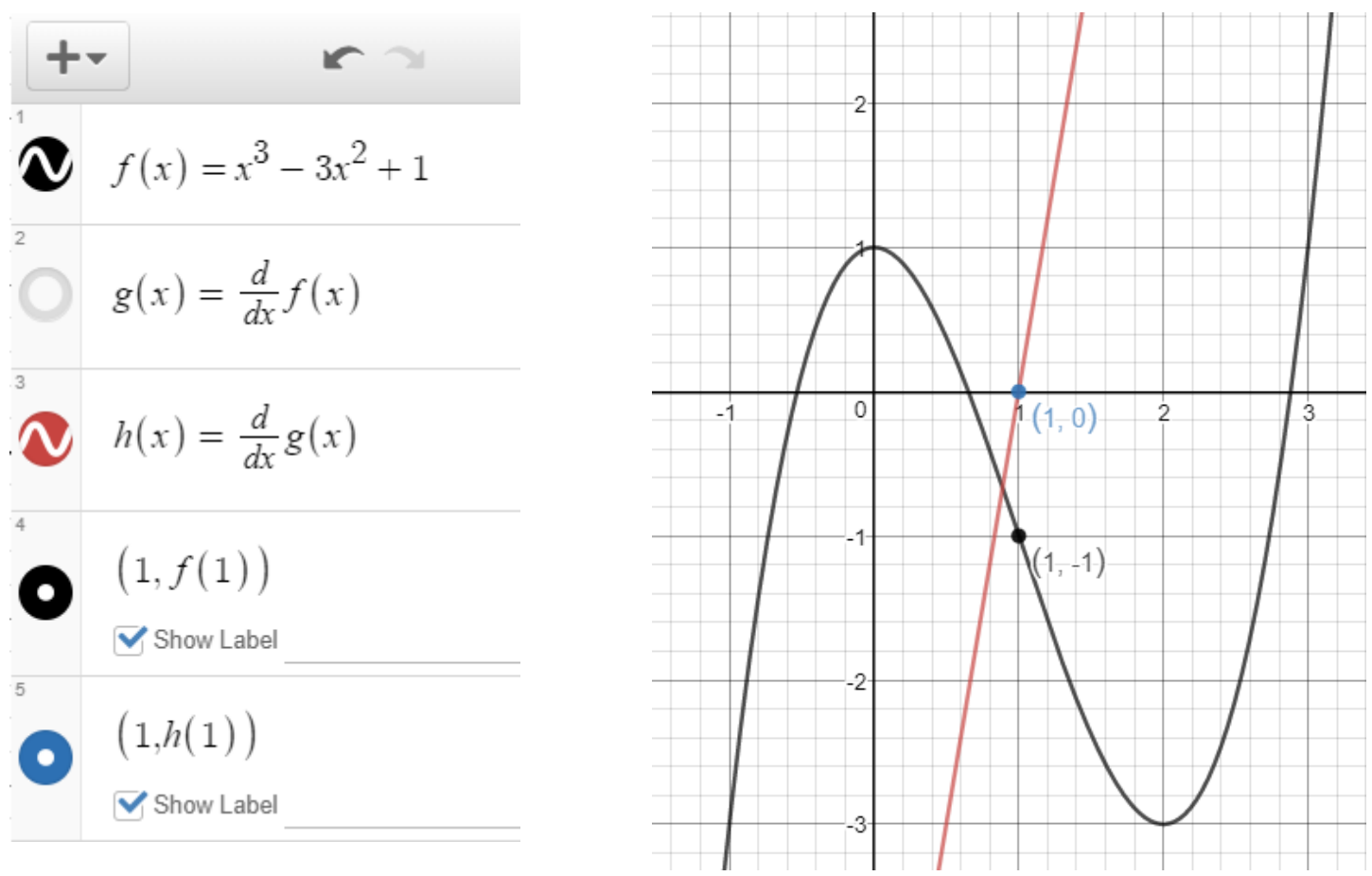

Fonte: elaborada pelos autores, 2017.

Na Figura 6 mostra-se o desenvolvimento das atividades do Quadro 2 do aluno B, com ajuda da visualização gráfica da Figura 5. 
Figura 6 - Resolução das atividades pelo aluno B

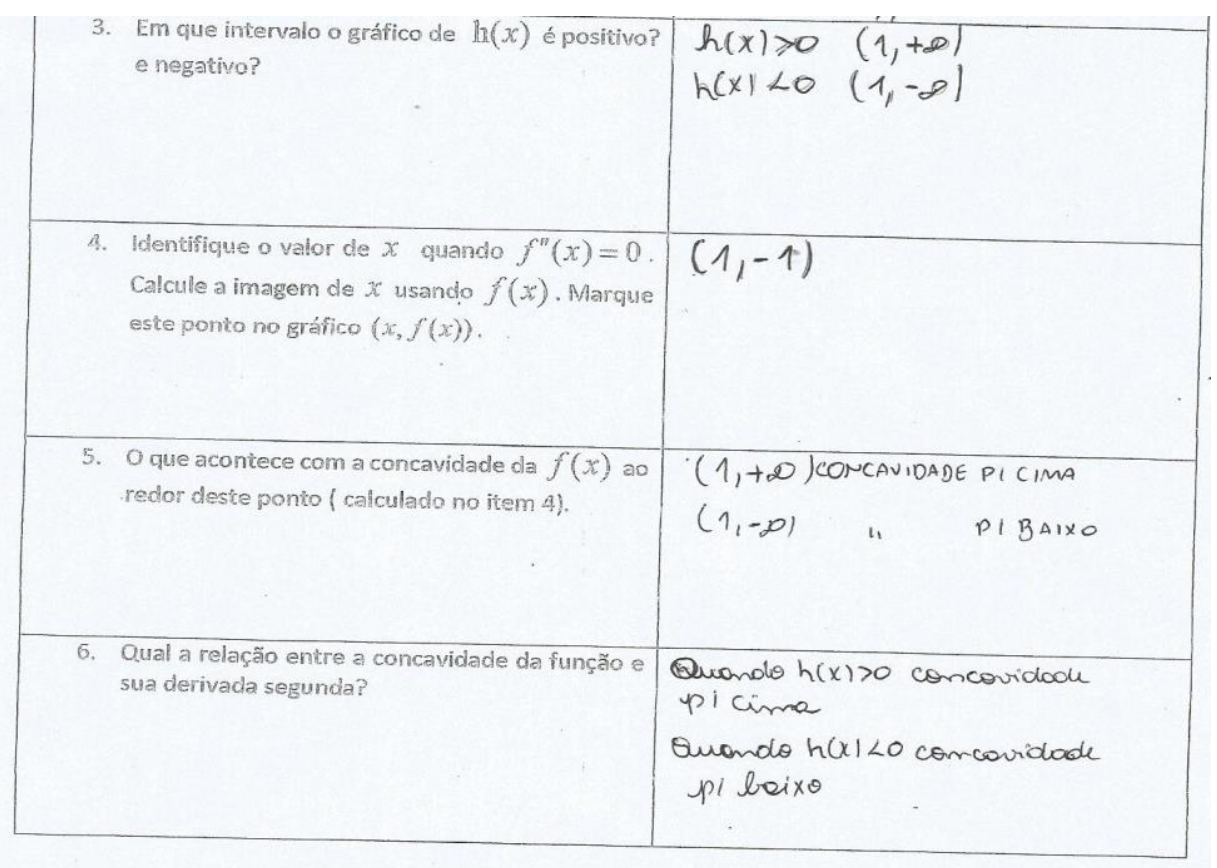

Fonte: atividades do aluno B.

Com essa atividade pretendeu-se que os alunos, após observação dos gráficos de $f$ e $h=f^{\prime \prime}$ , representados no mesmo sistema de eixos cartesianos (FIGURA 5), identificassem os intervalos dos sentidos das concavidades do gráfico de $f$ em função do sinal de $h=f^{\prime \prime}$ ao redor do ponto calculado em $f^{\prime \prime}(x)=0$, o que pode ser observado nas respostas do aluno $\mathrm{B}$ (FIGURA 6).

As respostas obtidas nessas atividades demonstram que a visualização auxilia no processo de construção de conceitos. Além disso, a linguagem utilizada pelos alunos, mesmo sendo coloquial em relação à Matemática, submete a possibilidade de se trabalhar de modo mais intuitivo, levando em consideração os conhecimentos anteriores dos alunos e a sua forma de representação e expressão matemática. De acordo com Henriques (2008), com o auxílio de softwares apropriados, a aprendizagem em Cálculo torna-se não apenas intuitiva, pois permite visualizar os procedimentos matemáticos de modo gráfico, numérico e algébrico. Acerca das contribuições do uso do software nas aulas de Cálculo, pode-se corroborar com Borba e Penteado (2001), que consideram a utilização de software ou tecnologia computacional uma possibilidade para a experimentação com conteúdos matemáticos, além de estimular a percepção visual do aluno.

Ao final das atividades foi aplicado um questionário, conforme o Quadro 3, e os alunos destacaram a importância do uso do Desmos para a visualização das funções e dos seus gráficos e o trabalho com derivadas. Seguem alguns comentários: 
A observação do gráfico facilita a compreensão e a correlacionar aspectos numéricos com o comportamento da função (Aluno C).

Através do software pode-se observar o gráfico e seus pontos de maneira mais clara (Aluno D).

Ajudou a visualizar os resultados, e torná-los menos abstratos (Aluno E).

Analisando os comentários, percebeu-se que a manipulação das imagens proporcionou a interpretação de propriedades matemáticas, validando as ideias de Reis e Júnior (2016), que destacam a importância da imagem visual para a compreensão dos conceitos envolvidos na disciplina de Cálculo e da interação desses conceitos com aspecto analítico. Ainda, segundo Costa e Souza Júnior (2007), com a utilização de tecnologias possibilita-se ao aluno construir ou, então, ressignificar conceitos já estudados que possam estar relacionados à visualização.

Os alunos também evidenciaram tais contribuições:

Possibilitou mais interatividade e contribuiu no aprendizado (Aluno E).

Torna o ensino menos monótono (Aluno F).

Tornou as aulas mais interativas (Aluno G).

Dentre as contribuições apontadas pelos alunos, essas são corroboradas por Marin (2013), ao apontar as vantagens do uso de tecnologias em sala de aula. Por meio destas, de acordo com o autor, os alunos aprendem mais rapidamente e melhor, pois os recursos tecnológicos - principalmente por meio do aspecto visual - agregam facilidade na compreensão de conteúdos que anteriormente pareciam abstratos. Ainda, proporciona-se a experimentação, mediante a qual pode-se buscar novas descobertas, observar propriedades, investigar, transformar, modificar e testar aquilo que antes somente era repassado ao aluno de maneira tradicional, automatizada e "fechada", uma vez que se constituía em conteúdo pronto, inquestionável. Corrobora-se também com Paranhos (2009), que evidencia a possibilidade de mudanças na qualidade do aprendizado dos alunos, pois eles se tornam mais participativos, exploradores e protagonistas do próprio aprendizado e, consequentemente, acompanham o curso com mais ânimo e comprometimento em relação aos processos de ensino e aprendizagem.

Da mesma forma, considerando as observações realizadas e as atividades desenvolvidas, é possível apontar significativos aspectos favoráveis ao uso do Desmos em sala de aula, destacando-se: 
a) A instituição de um ambiente mais dinâmico e interativo, principalmente pela interação tecnológica com a oportunidade de trabalho com o Desmos e, também, como o trabalho realizado em duplas.

b) O protagonismo dos acadêmicos, que assumiram um papel mais ativo em relação à construção do conhecimento envolvido na derivada de funções e na sua exploração gráfica, já que foram desafiados a realizar as atividades propostas para inferir e compreender o conteúdo elencado, em um trabalho de sequência inversa, ou seja, a prática antecedendo a teoria.

c) A possibilidade de compararação dos resultados obtidos numericamente com os gráficos das funções, oportunizando o entendimento de conceitos matemáticos antes vistos apenas algebricamente, como no caso do cálculo dos máximos e dos mínimos de uma função por meio do teste da derivada, que comumente é trabalhado algebricamente sem ser relacionado a seu aspecto gráfico.

d) Coparticipação da turma, com predisposição ativa, decorrente da simpatia pelas tecnologias e do aceite à provocação para realizar as atividades propostas antes mesmo de aula explicativa acerca do conteúdo.

e) A facilidade de manuseio do Desmos, apontada por todos os acadêmicos ao utilizá-lo, pois o programa possui interface amigável, layout simples e caráter didático.

\section{CONSIDERAÇÕES FINAIS}

Fica evidente que o trabalho com atividades de visualização gráfica utilizando o software Desmos, para o ensino de derivadas primeira e segunda e o comportamento de funções, proporciona importantes contribuições para os processos de ensino e de aprendizagem. Dessas, destacam-se: possibilidade de análise e comparação dos resultados obtidos algebricamente com os gráficos, ratificando aspectos citados por Arcavi (2003); alunos mais participativos devido à necessidade de realização das atividades para compreensão do conteúdo em estudo; ampliação da possibilidade de atividades variadas, nas quais os alunos podem trabalhar com diferentes representações, tais como tabelas, gráficos e expressões algébricas, de forma mais articulada - o que corrobora com Machado (2008).

Quanto ao trabalho docente ante o uso de uma tecnologia em sala de aula, cabe destacar a necessidade do conhecimento prévio sobre a tecnologia adequada e a metodologia a ser associada a essa ferramenta. Dessa forma, para fazer um trabalho significativo e coerente, a junção desses dois elementos é essencial, contribuindo, também, para um bom desempenho dos estudantes nas atividades propostas. Conforme Penteado (2000), é 
comum professores trabalharem em uma espécie de "zona de conforto", de modo que persistem fazendo o que rotineiramente a maioria faz, sem propor ou promover mudanças. Assim, julgam que tudo está sob controle e é previsível. Isso é observado, muitas vezes, no ensino tradicional, em que o professor acredita que transfere conhecimentos aos seus alunos, que, por sua vez, assumem papel passivo de quem recebe informações prontas e acabadas. Tal postura pode até parecer confortável para os professores que têm controle sobre a aula em meio à monotonia da baixa participação do principal agente da aprendizagem: o aluno. No entanto, quando se considera, no processo de ensinar, o desafio de mediar a aprendizagem, supõe-se metodologias ativas, foco no ser aprendente e nas inovações. Nesse sentido, é preciso considerar que, quando se opta por fazer algo novo, utilizar metodologias distintas e aproveitar recursos diferenciados, é necessário que o professor esteja preparado para sair dessa "zona de conforto" e entrar no que Penteado e Skovsmose (2008) denominaram como "zona de risco". Nesse contexto, com um docente que se arrisca e propõe o diferente, podem ocorrer novos fatos, uma vez que há flexibilidade, incerteza e imprevisibilidade, pois o professor deixa de ser o ator principal nos processos de ensino e aprendizagem e passa a ser um orientador e motivador em sala de aula.

Por conseguinte, o uso de atividades exploratórias associadas ao software Desmos demonstraram a possibilidade de substituir metodologias tradicionais por metodologias mais ativas, centradas no aluno e na preocupação com sua aprendizagem e seu aproveitamento, na disciplina de Cálculo I. Essa prática se mostra capaz de promover um ganho a todos, em especial por provocar a descoberta das soluções, em vez de trazê-las prontas e demonstrá-las. Reiterando, o resultado da prática de atividades exploratórias foi enriquecedor, de modo que se destaca um trabalho mais constante, envolvendo a exploração gráfica dos conceitos como auxílio para os cálculos, de maneira a desenvolver o senso crítico e analítico dos alunos. Pode-se inferir que a inclusão de recursos tecnológicos possibilitou alunos pensantes, questionadores e participativos, os quais foram constantemente desafiados a encontrar soluções matematicamente aceitáveis.

\section{REFERÊNCIAS}

ALLEVATO, Norma S. G. Utilizando animação computacional no estudo de funções. Revista de Ensino de Ciências e Matemática, São Paulo, v. 1, n. 2, p. 111-125, 2010. Disponível em: <http://revistapos.cruzeirodosul.edu.br/index.php/rencima/article/view File/13/15>. Acesso em: 20 mar. 2017.

ALVARENGA, Karly B.; DORR, Raquel C.; VIEIRA, Vanda D. O ensino e a aprendizagem de Cálculo Diferencial e Integral: características e interseções no centro-oeste brasileiro. Revista Brasileira de Ensino Superior, Passo Fundo, v. 2, n. 4, p. 46-57, 2016. Disponível 
em: <https://seer.imed.edu.br/index.php/REBES/article/download/1518/1069>. Acesso em: 24 mar. 2017.

ANDRADE, Pedro F. Aprender por Projetos, Formar Educadores. In: FORMAÇÃO DE EDUCADORES PARA O USO DA INFORMÁTICA NA ESCOLA. Campinas, SP: UNICAMP/NIED, 2003. Disponível em: <http://www.nied.unicamp.br/?q=content/forma\%C3\%A7\%C3\%A3 o-de-educadores-para-o-uso-da-inform\%C3\%A1tica-na-escola>. Acesso em: 23 maio 2017.

ARCAVI, Abraham. The Role of Visual Representations in the Learning of Mathematics. In: EDUCATIONAL STUDIES IN MATHEMATICS. Proceedings... n. 52, p. 54-80, 2003. Disponível em: <https://files.eric.ed.gov/fulltext/ED466382.pdf>. Acesso em: 20 mar. 2017.

BORBA, Marcelo de C.; PENTEADO, Miriam G. Informática e Educação Matemática. Belo Horizonte: Autêntica, 2001. 98 p.

BORBA, Marcelo de C.; VILLARREAL, Monica. E. Humans-With-Media and the Reorganization of Mathematical Thinking: information and communication technologies, modeling, experimentation and visualization. New York: Springer, 2005. v. 39.

BRASIL. Ministério da Educação e do Desporto. Secretaria de Educação Fundamental. Parâmetros curriculares nacionais terceiro e quarto ciclos do ensino fundamental: introdução aos parâmetros curriculares nacionais. Brasília, DF: MEC/SEF, 1998.

CHIAPPINI, Giampaulo; BOTTINO, Rosa M. Visualisation in teaching-learning mathematics: the role of the computer. In: PROCEEDINGS OF GRAPHICS AND VISUALIZATION EDUCATION. Proceedings... Coimbra: Portugal. 1999. p. 143-147. Disponível em: <http://old.siggraph.org/education/conferences/GVE99/papers/GVE99.Proceedings.pdf> . Acesso em: 1 abr. 2017.

COSTA, Ivo M. da; SALVADOR, José A. Ensino de Cálculo Diferencial e Integral: Experiências no DM - UFSCar. In: ENCONTRO PAULISTA DE EDUCAÇÃO MATEMÁTICA, VII. Anais... São Paulo, SP, 2004. Disponível em: <http://www.sbempaulista.org.br>. Acesso em: 1 mar. 2017.

COSTA, Patricia O.; SOUZA JÚNIOR, Arlindo J. Tecnologia da informação e comunicação no ensino de Cálculo. FAMAT em Revista, Uberlância, n. 9, p. 431-440, 2007. Disponível em:

<http://www.portal.famat.ufu.br/sites/famat.ufu.br/files/Anexos/Bookpage/Famat_revi sta_09_sala_09.pdf>. Acesso em: 1 mar. 2017.

FAINGUELERNT, Estela K. Educação Matemática: representação e construção em geometria. Porto Alegre: Artes Médicas Sul, 1999. 
FLORES, Cláudia R.; WAGNER, Débora R.; BURATTO, Ivone C. F. Pesquisa em visualização na Educação Matemática: conceitos, tendências e perspectivas. Revista Educação Matemática e Pesquisa, São Paulo, v. 14, n. 1, p. 31-45, 2012. Disponível em: <https:// revistas.pucsp.br/index.php/emp/article/view/8008/6827>. Acesso em: 22 mar. 2017.

HENRIQUES, Afonso. Estudo de Integrais Múltiplas no Ambiente Computacional Maple. In: I ENCONTRO ALAGOANO DE EDUCAÇÃO MATEMÁTICA. Anais... Arapiraca, 2008. Disponível em: <http://www.uesc.br/cursos/graduacao/bacharelado/matematica/minic urso_sobre_integrais_multiplas_com_maple.pdf>. Acesso em: 3 abr. 2017.

JUNIOR, José C. M. Ensino de derivadas em Cálculo l: aprendizagem a partir da visualização com o uso do GeoGebra. 2015. 123 f. Dissertação (Mestrado em Educação Matemática) - Instituto de Ciências Exatas e Biológicas, Universidade Federal de Ouro Preto, Ouro Preto, 2015. Disponível em: <http://www.ppgedmat.ufop.br/arquivos/diss ertacoes_2015/Jose\%20Cirqueira\%20Martins\%20Junior.pdf>. Acesso em: 15 abr. 2017.

JUNIOR, José F. G.; BESSA, Vagner R. de; CEZANA, Miguel J. Um estudo sobre o baixo índice de aprovação nas disciplinas de Cálculo da Universidade Federal de Viçosa Campus Rio Paranaíba. Revista lluminart, Sertãozinho, ano VII, n. 13, dez. 2015. Disponível em: <http://revistailuminart.ti.srt.ifsp.edu.br/index.php/iluminart/article/view /270/265>. Acesso em: 30 mar. 2017.

MACHADO, Rosa M. A visualização na resolução de problemas de Cálculo Diferencial e Integral no ambiente computacional MPP. 2008. 287 f. Tese (Doutorado em Educação) Universidade Estadual de Campinas, Campinas, 2008. Disponível em: $<$ http://repositorio.unicamp.br/bitstream/REPOSIP/251984/1/Machado_RosaMaria_D.p df>. Acesso em: 30 maio 2017.

MARIN, Douglas. O uso de tecnologia de informação e comunicação nas aulas de Cálculo: vantagens e desvantagens. In: ENCONTRO NACIONAL DE EDUCAÇÃO MATEMÁTICA: Educação Matemática: Retrospectivas e Perspectivas: XI ENEM, Curitiba/PR. Anais... Curitiba, 2013. Disponível em: <http://sbem.web1471.kinghost.net/anais/XIENEM/pdf/ 1331_649_ID.pdf>. Acesso em: 2 fev. 2017.

NASCIMENTO, Carlos H. da S.; LOPES, Daniella O.; TEIXEIRA, Paulo C. M. Utilização do software Maple no problema de Cálculo: Modelagem Matemática de um volume de revolução. In: ENCONTRO NACIONAL DE EDUCAÇÃO MATEMÁTICA: Educação Matemática: Retrospectivas e Perspectivas: XI ENEM, Curitiba/PR. Anais... Curitiba, 2013. Disponível em: <http://sbem.web1471.kinghost.net/anais/XIENEM/pdf/1986_978_ID. pdf>. Acesso em: 30 jan. 2017.

PARANHOS, Marcos de M. Geometria dinâmica e o cálculo diferencial e integral. 2009. 112 f. Dissertação (Mestrado em Educação) - Pontifícia Universidade Católica de São Paulo, São Paulo, 2009. Disponível em: <https://tede2.pucsp.br/handle/handle/11408>. Acesso em: 30 mar. 2017. 
PENTEADO, Miriam G. Possibilidades para a formação de professores de Matemática. In: PENTEADO, Miriam G.; BORBA, M. C. (Org.). A Informática em Ação: formação de professores, pesquisa e extensão. São Paulo: Olho Dágua, 2000. p. 23-34.

PENTEADO, Miriam G.; SKOVSMOSE, Ole. Riscos trazem possibilidades. In: SKOVSMOSE, Ole. Desafios da reflexão em educação matemática crítica. Campinas: Papirus, 2008. p. 41-50.

PONTE, João P.; BROCARDO, Joana; OLIVEIRA, Hélia. Investigação Matemática na sala de aula. Belo Horizonte: Autêntica, 2006.

PRESMEG, Norma. Research on visualization in learning and teaching mathematics: emergence from Psychology. In: GUTIÉRREZ, A.; BOERO, P. Handbook Of Research On The Psychology Of Mathematics Education. Rotterdam: Sense, 2006. p. 205-235. Disponível em: $\quad$ http://www.kaputcenter.umassd.edu/downloads/symcog/bib/pmeVisualization FinalAPA.pdf >. Acesso em: 1 abr. 2017.

RAFAEL, Rosane C.; ESCHER, Marco A. Evasão, baixo rendimento e reprovações em Cálculo Diferencial e Integral: Uma questão a ser discutida. In: ENCONTRO MINEIRO DE EDUCAÇÃO MATEMÁTICA, 7, 2015. Anais... Juiz de Fora, 2015. Disponível em: <http://www.ufjf.br/emem/files/2015/10/EVAS\%C3\%83O-BAIXO-RENDIMENTO-EREPROVA\%C3\%87\%C3\%95ES-EM-C\%C3\%81LCULO-DIFERENCIAL-E-INTEGRAL-UMAQUEST\%C3\%830-A-SER-DISCUTIDA-2.pdf>. Acesso em: 17 mar. 2017.

REIS, Frederico da S.; JÚNIOR, José C. M. As contribuições da visualização proporcionada pelo Geogebra à aprendizagem de funções derivadas em Cálculo I. In: XII ENCONTRO NACIONAL DE EDUCAÇÃO MATEMÁTICA: Educação Matemática na Contemporaneidade: desafios e possibilidades (XII ENEM). Anais... São Paulo, 2016. Disponível em: <http://www.sbembrasil.org.br/enem2016/anais/pdf/8057_3666_ID.pdf>. Acesso em: 30 mar. 2017.

RÖSKEN, Bettina; ROLKA, Katrin. A picture is worth a 1000 words: the role of visualization in mathematics learning. In: PROCEEDINGS 30TH CONFERENCE OF THE INTERNATIONAL GROUP FOR THE PSYCHOLOGY OF MATHEMATICS EDUCATION, PME. Proceedings... Prague, 2006. p. 457-464. Disponível em: <https://files.eric.ed.gov/fulltex t/ED496934.pdf\#page=465>. Acesso em: 24 mar. 2017.

SANTOS, Alessandra H. Um Estudo Epistemológico da Visualização Matemática: o acesso ao conhecimento matemático no ensino por intermédio dos processos de visualização. 2009. 98 f. Dissertação (Mestrado em Educação Matemática) - Setor de Ciências Exatas, Universidade Federal do Paraná, Curitiba, 2009. Disponível em: <http://www.exatas.ufpr. $\mathrm{br} /$ portal/ppgecm/wp-

content/uploads/sites/27/2016/03/045_AlessandraHendidosSanto s.pdf>. Acesso em: 24 mar. 2017. 
SILVA, Jayro F. Questões metodológicas do ensino de Cálculo Diferencial e Integral I. 1994. 148 f. Dissertação (Mestrado em Educação) - Faculdade de Educação, Universidade Federal do Ceará, Fortaleza, 1994.

STEWART, James. Cálculo: volume 1. São Paulo: Cengage Learning, 2017.

ZIMMERMANN, Walter; CUNNINGHAM, Steve. Editors' introduction: What is mathematical visualization? In: ZIMMERMANN, Walter. Visualization In Teaching And Learning Mathematics. Mathematical Association of America, Washington, D.C., 1991.

p. 1-8. Disponível em:

$<$ https://pdfs.semanticscholar.org/4633/9bd5e52c9f6785b70ef1e

62812f5f02b1ec7.pdf>. Acesso em: 2 abr. 2017.

\section{Gisele Scremin}

Mestranda do curso de pós-graduação em Ensino de Ciências Exatas, pela Universidade do Vale do Taquari (UNIVATES), de Lajeado/RS. Possui especialização em Tecnologias da Informação e Comunicação aplicadas à Educação pela Universidade Federal de Santa Maria (2017), graduação em Matemática pela Universidade Regional Integrada do Alto Uruguai e das Missões (2009).

gisele23scremin@gmail.com

\section{Marli Teresinha Quartieri}

Doutora em Educação pela Universidade Vale do Rio dos Sinos/RS. É professora titular da UNIVATES. Tem experiência na área de Ensino de Matemática. Atua nos cursos de graduação e pós-graduação (Mestrado em Ensino de Ciências Exatas e Mestrado e Doutorado em Ensino).

mtquartieri@univates.br

\section{Eniz Conceição Oliveira}

Doutora em Química pela Universidade Federal do Rio Grande do Sul. É professora titular da UNIVATES. Tem experiência na área de Ensino de Química. Atua nos cursos de graduação e pós-graduação (Mestrado e Doutorado em Ambiente e Desenvolvimento, Mestrado em Ensino de Ciências Exatas e Mestrado e Doutorado em Ensino).

eniz@univates.br

\section{Jorge Luis Palacios Felix}

Doutor em Engenharia Mecânica pela Universidade Estadual de Campinas (UNICAMP) e pósdoutor em Matemática Aplicada pela Universidade Estadual Paulista (UNESP). É professor adjunto da Universidade Federal da Fronteira Sul (UFFS), campus Cerro Largo. Atua nos cursos de graduação e pós-graduação (Mestrado em Ambiente e Tecnologias Sustentáveis). jorge.felix@uffs.edu.br 